\title{
Detection of Colorectal Cancer Hepatic Metastases with Contrast-Enhanced Ultrasound: Comparison with Conventional B-Mode Ultrasound
}

\author{
S.D. Yarmenitis A. Karantanas A. Bakantaki Y. Papantoniou N. Gourtsoyiannis \\ Department of Radiology, University of Crete, Faculty of Medicine, Heraklion, Greece
}

\section{Key Words}

Colorectal cancer/imaging $\cdot$ Liver neoplasms/

ultrasonography $\cdot$ Contrast media $\cdot$ Metastatic disease/

diagnosis

\begin{abstract}
The aim of this study was to assess whether contrast-enhanced ultrasound (CE-US) could provide improved diagnostic information in detecting liver metastases from colorectal cancer as compared to B-mode non-enhanced ultrasound (B-US). 32 patients (M/F 23/9, age range 48-82 years, mean 58.2 years) under chemotherapy for colorectal cancer were examined with B-US and CE-US using a secondgeneration ultrasound contrast agent and a dedicated protocol for contrast detection. The presence of focal liver lesions along with the number, size, pre- and post-contrast sonographic features were recorded digitally. Lesion conspicuity with a three-grade scoring scale was performed on both techniques and contrast intensity measurements were calculated for each focal lesion. CE-US detected $17 \%$ more metastases in patient-by-patient and lesion-by-lesion analysis. A statistically significant difference was found between the scoring mean values with regard to conspicuity of the lesions. Accurate characterization of the liver lesions was achievable only with contrast-enhanced technique. A quantitative contrast intensity measurement method confirmed
\end{abstract}

the invariably washing-out vascular pattern in all metastases at sinusoidal-parenchymal liver phase. In conclusion, CE-US is superior to B-US and provides an effective tool in the investigation of colorectal cancer liver metastases.

Copyright $\odot 2007$ S. Karger AG, Basel

\section{Introduction}

The early detection and precise characterization of liver metastases from colorectal carcinoma has become a crucial issue in treatment planning and is also considered to be important for improved therapeutic outcomes. In most of colorectal cancer patients, conventional B-mode non-enhanced ultrasound (B-US) is the first-line imaging modality for detecting liver metastases. However, US in the detection of focal liver lesions presents a sensitivity ranging from 40 to $80 \%$ according to the diameter of lesions and to the experience of the sonologist $[1,2]$.

The introduction of ultrasound contrast agents and the advances in ultrasound apparatuses that are now able to detect these agents in real time have improved the overall accuracy of US in the characterization of focal liver lesions $[3,4]$, in liver metastases detection [5-10] and in monitoring the effect of therapy in liver metastases [11].

\section{KARGER \\ Fax +41613061234 \\ E-Mail karger@karger.ch}

www.karger.com (c) 2007 S. Karger AG, Basel

0257-2753/07/0251-0086\$23.50/0

Accessible online at:

www.karger.com/ddi
A. Karantanas, $\mathrm{MD}, \mathrm{PhD}$

Department of Radiology, University Hospital

GR-711 10 Stavrakia/Heraklion (Greece)

Tel. +30 2810392 541, Fax +30 2810542095

E-Mail apolsen@yahoo.com or karantanas@med.uoc.gr 

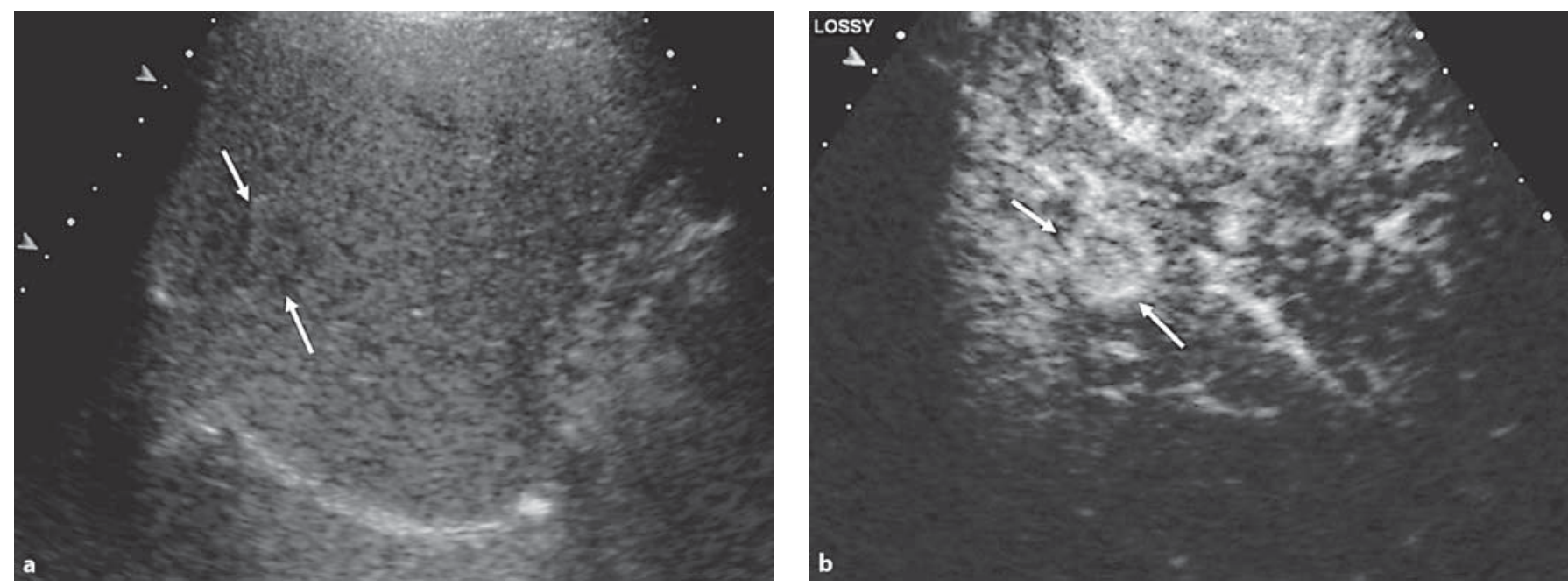

Fig. 1. A 55-year-old male patient with history of rectal carcinoma under chemotherapy and regular imaging follow-up. a The 2-year non-enhanced B-US discloses a target-like liver metastatic lesion with isoechoic center and hypoechoic margin (arrows). b CE-US at post-injection arterial liver phase shows the hypervascular characteristics of the lesion (arrows). c In the sinusoidal-parenchymal liver phase CE-US, there is contrast agent washout and the lesion appears hypovascular against the surrounding liver parenchyma (arrows).

A recent study [12] compared contrast-enhanced ultrasound (CE-US) with B-US and contrast-enhanced spiralCT in metastatic, of any primary cancer, disease of the liver. CE-US was found to have an $83 \%$ sensitivity, $84 \%$ specificity and a high accuracy as shown by a 0.929 ROC under the curve analysis.

Since the introduction of CE-US, all published data on the investigation of hepatic metastatic disease refer to hepatic metastases secondary to any primary. To our knowledge, there are no reports in the English literature that assess the performance of this new method exclusively on the colorectal cancer liver metastases.

Our purpose in this study was to evaluate whether the detection and characterization of liver metastases from colorectal carcinoma can be improved by using CE-US, as compared with non-enhanced B-US.

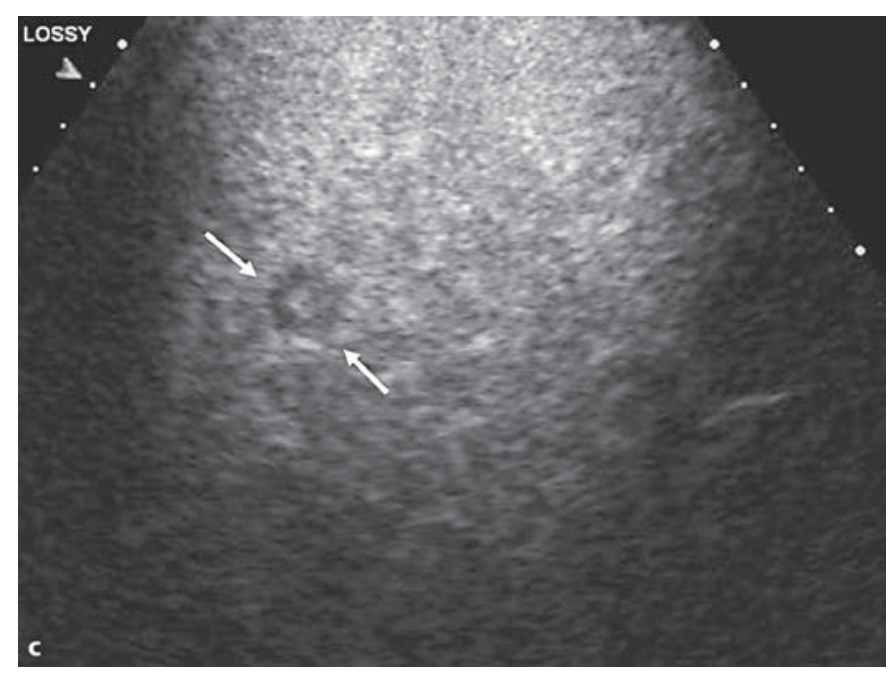

\section{Materials and Methods}

Over a period of 1 year, 32 consecutive patients (23 males and 9 females, age range $48-82$ years, mean age 58.2 years) under chemotherapy for a diagnosed colorectal carcinoma, were included in this study. An overall informed consent for diagnostic and therapeutic procedures, including the pre- and post-contrast ultrasound studies of the liver, was promptly provided to the referring physicians. Approval from the ethics committee of our institution was not needed since CE-US of the liver is among the labeled indications of the specific US contrast agent that was used in this study.

All patients were examined with a Siemens, Acuson Sequoia 512 (Signature II Version 8) ultrasound unit equipped with a $4 \mathrm{C1}$, $2-4-\mathrm{MHz}$ curved array abdominal transducer. A non-enhanced B-US investigation preceded all CE-US studies. Tissue harmonic and spatial compounding software were used as a standard preset for the B-US scans in order to reduce noise and speckle artifacts in an effort to increase baseline lesion conspicuity. The contrast- 

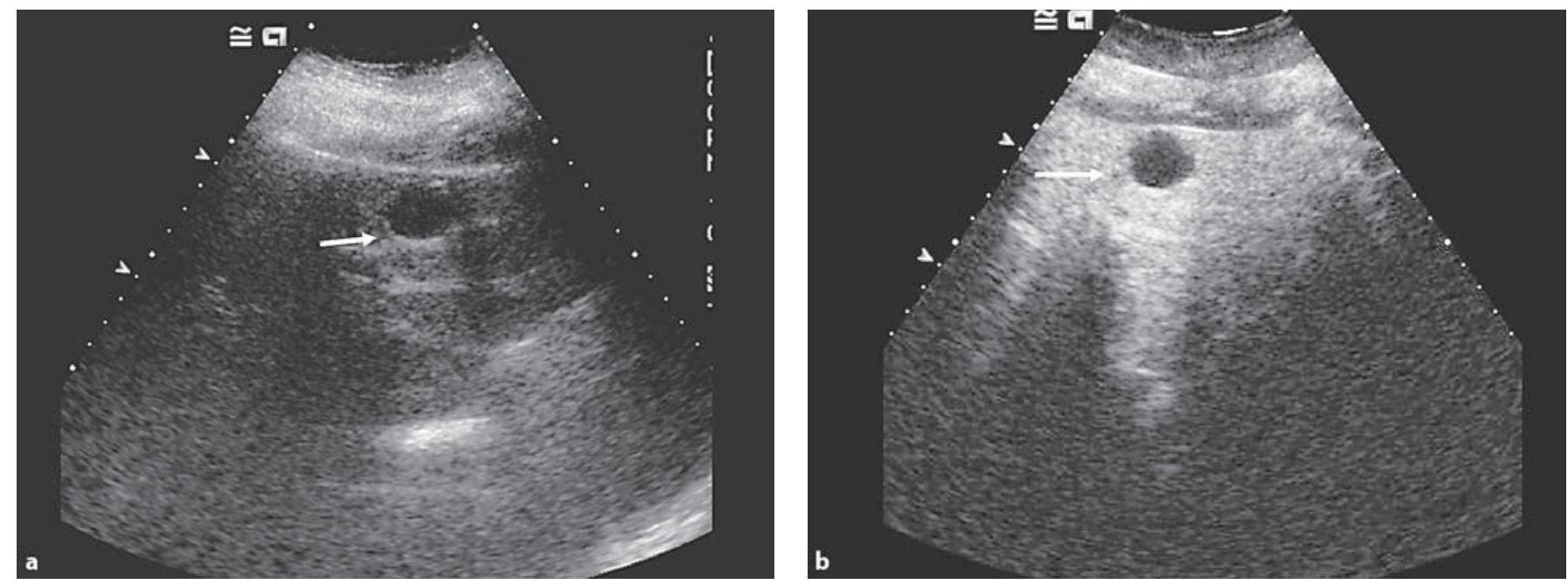

Fig. 2. A parenchymal liver cyst at non-enhanced B-US (a) and CE-US (b) appears totally anechoic with sharp borders and posterior echo enhancement (arrow).
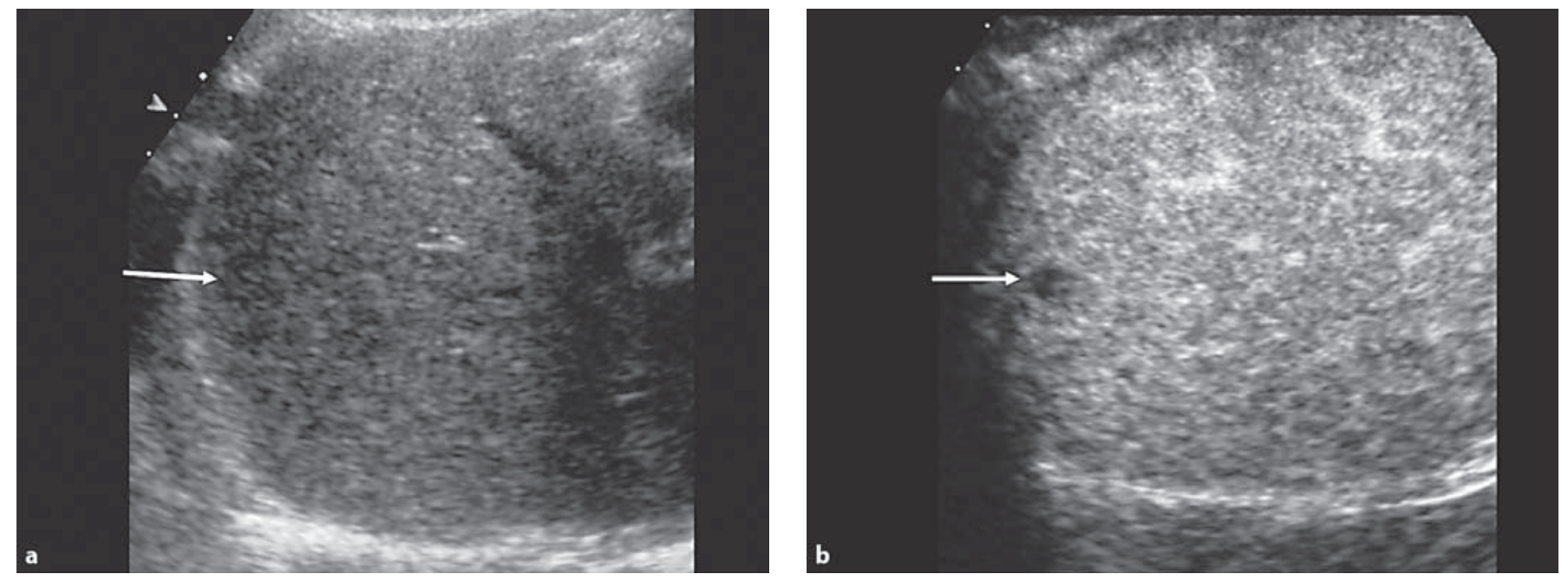

Fig. 3. Patient with left colon carcinoma, operated 3 years prior to imaging. a The non-enhanced B-US of right liver lobe shows a suspected focal liver lesion (arrow) with extremely poor conspicuity and lack of tissue reflectivity differentiation. CE-US (b) reveals a subcentimeter (c) hypovascularized colorectal cancer metastasis (arrow). Detection and characterization was impossible with baseline US.

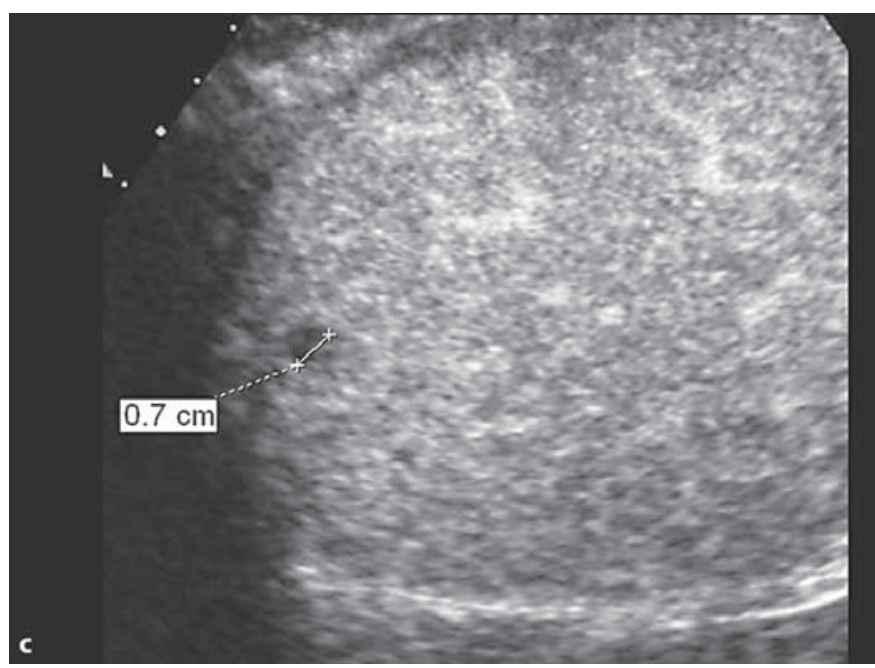


specific scan protocol was based on the Contrast Pulse Sequencing (CPS) software for low mechanical index contrast detection.

SonoVue Bracco Sulfur hexafluoride (SF6) microbubbles were used as ultrasound contrast agent at a standard intravenous dose of $2.4 \mathrm{ml}$. A supplementary dose of $2.4 \mathrm{ml}$ was occasionally administered to ascertain diagnostic information in some cases. A third dose was never required. The perfusion process was evaluated in real time, utilizing CPS mode ability to interchange between contrast only, mixed or B-mode only imaging. All studies were recorded with the use of dedicated software in digital video sequences lasting for $3 \mathrm{~min}$ at least. Off-line workstation reading of the recorded ultrasound examinations followed thereafter by two on-site radiologists (S.D.Y. with 20 and 6 years of ultrasound and contrast studies experience, and A.B. with 15 and 3 years experience respectively).

From the review of the digital recordings, each case was assessed for the presence or not of focal liver lesions. Thereafter the lesion number, size and characteristic patterns on B-US and on CE-US were recorded and a three-grade lesion conspicuity score was applied on both techniques as follows: grade 3 for lesions that showed optimum conspicuity, grade 2 for suboptimal conspicuity and grade 1 for poor or no conspicuity at all. The grading was done in a side-by-side fashion and final scoring decision was reported by consensus. Statistical analysis was performed with Mann-Whitney test for the lesion conspicuity grading. The CEUS features for diagnosing a metastasis were those of a hyper- or hypoenhancing lesion at 15-20 s post-contrast injection followed by a clear washout of the contrast agent at $60-90$ s post-injection resulting in a hypovascular focus against hyperenhanced neighboring liver parenchyma (fig. 1) [13].

Hemangiomas were diagnosed when a lesion showed either gradual slow peripheral centripetal enhancement or rapid initial overall enhancement but with continuous persistent hyperenhancement throughout the study.

Contrast intensity measurement was performed for each detected lesion using the Merge Efilm Workstation Version 1.8 software by defining regions of interest (ROI). Arbitrary intensity values were taken from each ROI at 20 and 60 s post-contrast injection.

The final diagnosis of those lesions considered as metastatic was established with biopsy or multimodality imaging assessment with characteristic findings on contrast-enhanced CT or MR imaging including progressive enlargement or subsequent reduction in size following chemotherapy. Dynamic contrast-enhanced CT or MR imaging findings reported as showing diagnostic features of hemangioma with no change at follow-up of longer than 1 year.

\section{Results}

No lesions were found in 13 patients at both B-US and CE-US. In 2 of these individuals, CE-CT revealed 1 and 2 subcentimeter lesions respectively. One of these turned out to be a metastasis in a subsequent follow-up CT scan, whereas the others remained unchanged. It was not feasible to define with contrast-enhanced helical CT whether these two lesions were tiny cysts or hemangiomas.
Table 1. Number of detected lesions and corresponding lesion conspicuity score in each one of the US techniques (non-marked cases correspond to metastatic lesions cases)

\begin{tabular}{|c|c|c|c|c|}
\hline $\begin{array}{l}\text { Patients' } \\
\text { initials }\end{array}$ & $\begin{array}{l}\text { Number of } \\
\text { lesions, non- } \\
\text { enhanced US }\end{array}$ & $\begin{array}{l}\text { Number } \\
\text { of lesions } \\
\text { CE-US }\end{array}$ & $\begin{array}{l}\text { Conspicuity score } \\
\text { non-enhanced } \\
\text { US mean: } 2.23^{+}\end{array}$ & $\begin{array}{l}\text { Conspicuity } \\
\text { score, CE-US } \\
\text { mean: } 2.82^{+}\end{array}$ \\
\hline $\mathrm{HS}^{* *}$ & 1 & 1 & 3 & 3 \\
\hline PI & 2 & 2 & 2 & 3 \\
\hline MS & 0 & 2 & 1 & 3 \\
\hline $\mathrm{ME}^{* *}$ & 3 & 4 & 3 & 3 \\
\hline $\mathrm{MM}^{*}$ & 0 & 0 & & \\
\hline $\mathrm{KE}$ & $>5$ & $>10$ & 2 & 3 \\
\hline $\mathrm{KA}^{*}$ & 0 & 0 & & \\
\hline ZG & 0 & 1 & 1 & 3 \\
\hline $\mathrm{BZ}^{*}$ & 0 & 0 & & \\
\hline $\mathrm{PN}$ & 1 & 2 & 1 & 2 \\
\hline $\mathrm{MG}^{*}$ & 0 & 0 & & \\
\hline $\mathrm{BG}^{*}$ & 0 & 0 & & \\
\hline $\mathrm{BM}^{*}$ & 0 & 0 & & \\
\hline $\mathrm{SK}^{*}$ & 0 & 0 & & \\
\hline $\mathrm{ZN}^{*}$ & 0 & 0 & & \\
\hline $\mathrm{PK}^{* *}$ & 2 & 2 & 2 & 2 \\
\hline $\mathrm{KM}^{*}$ & 0 & 0 & & \\
\hline $\mathrm{KH}^{* *}$ & 2 & 2 & 3 & 3 \\
\hline $\mathrm{TK}^{\mathrm{a}}$ & 0 & 1 & 1 & 3 \\
\hline $\mathrm{PE}^{\mathrm{a}, * *}$ & 2 & 2 & 2 & 3 \\
\hline $\mathrm{KF}^{*}$ & 0 & 0 & & \\
\hline $\mathrm{TN}$ & $>10$ & $>10$ & 3 & 3 \\
\hline $\mathrm{HD}$ & 1 & 1 & 3 & 3 \\
\hline $\mathrm{KA}^{*}$ & 0 & 0 & 1 & $1^{\mathrm{b}}$ \\
\hline MS & 1 & 1 & 3 & 3 \\
\hline $\mathrm{MM}^{*}$ & 0 & 0 & 3 & 3 \\
\hline $\mathrm{GK}^{\mathrm{a}}$ & 1 & 1 & 2 & 3 \\
\hline KK & 3 & 3 & 3 & 3 \\
\hline $\mathrm{HN}^{*}$ & 0 & 0 & 3 & 3 \\
\hline $\mathrm{GH}$ & 0 & 2 & 1 & 3 \\
\hline IV & 3 & 3 & 3 & 3 \\
\hline $\mathrm{LE}^{\mathrm{c}}$ & 3 & 3 & 3 & 3 \\
\hline
\end{tabular}

Conspicuity score: 1 = poor; 2 = suboptimal; 3 = optimal lesion conspicuity.

* No lesions found. ${ }^{* *}$ Parenchymal cysts.

${ }^{a}$ Hemangioma. ${ }^{b}$ Fatty liver. ${ }^{c}$ Calcified metastases (primary mucinous adenocarcinoma). ${ }^{+} \mathrm{p}=0.001$ : statistical significance of the difference of conspicuity score mean values, between the nonenhanced and contrast-enhanced ultrasound.

Nineteen individuals showed focal lesions. Both ultrasound techniques revealed ten parenchymal cysts in $5 \mathrm{pa}-$ tients ranging from 0.6 to $3.6 \mathrm{~cm}$ (fig. 2). Two of them were subcentimeter lesions $(0.6$ and $0.7 \mathrm{~cm})$. Two hyperechoic solitary hemangiomas 1.2 and $2.7 \mathrm{~cm}$ were detected by both US modes in 2 patients. A third hypoechoic $1-\mathrm{cm}$ lesion in another patient, in B-US, turned out to be 
Table 2. Size and pre- and post-contrast echographic patterns of lesions

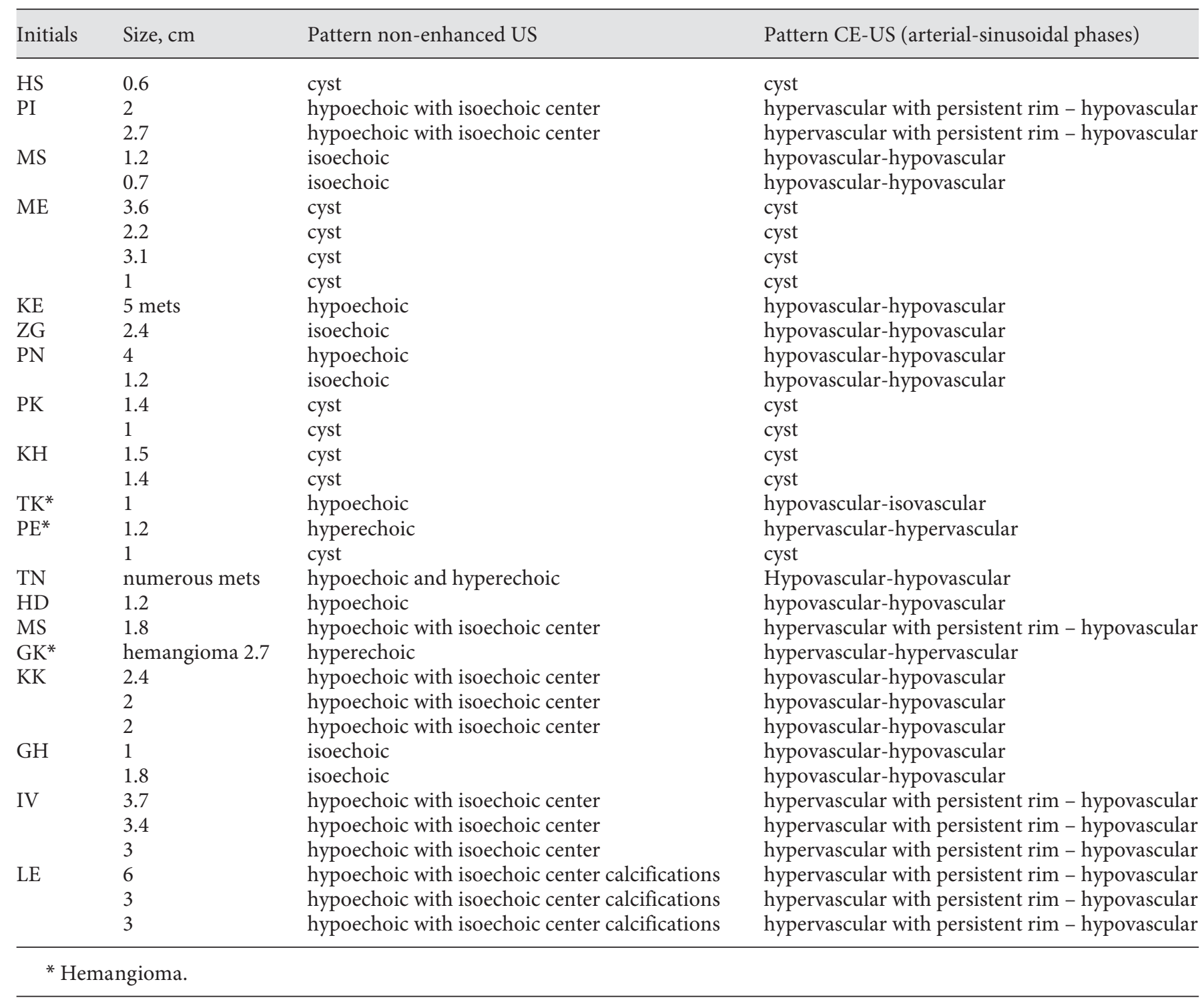

a hemangioma on CE-US. One cyst and one hemangioma coexisted in 1 patient.

A total of $12(37.5 \%)$ patients were diagnosed as having hepatic metastases. Eleven of them had 1-5 lesions and 1 presented with diffuse metastatic disease. Ten patients (83\%) were found to have metastases with both US techniques, whereas $2(17 \%)$ were diagnosed only with CEUS. Of these 2 individuals, 1 had a solitary isoechoic metastasis of $2.4 \mathrm{~cm}$ and the other had two isoechoic metastases of 0.7 and $1.2 \mathrm{~cm}$ (fig. 3).

On a lesion-by-lesion basis and after excluding 1 patient with diffuse disease, a total of 32 metastatic foci
(59\%) were localized by both US techniques, whereas 9 metastatic foci (17\%) were detected only with CE-US (tables 1,2 ).

Various patterns of echogenicity were found in the baseline non-enhanced US for the metastatic foci (ill-defined isoechoic $\mathrm{n}=6$, hypoechoic $\mathrm{n}=4$, and target-like hypoechoic with isoechoic center $n=12$ ). On CE-US all metastatic lesions were invariably hypovascular at the parenchymal-sinusoidal phase at $40-90$ s post-contrast injection. However, nine lesions showed a hypervascular activity during the arterial phase, $15-25 \mathrm{~s}$ post-contrast injection, and all were of the target-like 'hypoechoic with 
Table 3. Contrast intensity measurements of metastatic foci and hemangiomas at arterial and sinusoidal liver phases

\begin{tabular}{lcl}
\hline Initials & $\begin{array}{l}\text { Intensity values } \\
\text { arterial phase }\end{array}$ & $\begin{array}{l}\text { Intensity values sinusoidal- } \\
\text { parenchymal phase }\end{array}$ \\
\hline $\mathrm{PI}$ & 98.5 & 77.5 \\
$\mathrm{MS}$ & 100 & 83.2 \\
& 77.7 & 68.9 \\
$\mathrm{ZG}$ & $* *$ & 87 \\
$\mathrm{PN}$ & 97.4 & 72.6 \\
& 93.1 & 60.4 \\
$\mathrm{TK}$ & 115.2 & 57.3 \\
$\mathrm{PE}$ & 31.6 & 57.4 \\
$\mathrm{HD}$ & 153.2 & 163.5 \\
$\mathrm{MS}$ & 57 & 42 \\
$\mathrm{GK}$ & 117.6 & 65.4 \\
$\mathrm{KK}$ & 65.8 & 87.3 \\
& 109.3 & 72.2 \\
$\mathrm{GH}$ & 99.3 & 73 \\
& 98 & 67.5 \\
$\mathrm{IV}$ & 70 & 58 \\
& 74 & 63 \\
$\mathrm{LE}$ & 97.1 & 67.5 \\
& 92 & 68 \\
& 95.6 & 60.7 \\
\hline
\end{tabular}

Intensity values are arbitrary measurements of contrast intensity calculated by Merge Efilm Workstation Version 1.8 by defining regions of interest (ROI) at the specific lesions at 20 and $60 \mathrm{~s}$ post-injection.

* Hemangioma. ${ }^{* *}$ Arterial phase missing.

isoechoic center' pattern at the baseline US. The rest of the lesions were of the hypovascular type during the arterial phase. It is interesting that this hypovascularity at the arterial phase was presented by all as the ill-defined 'isoechoic' type of metastases that were not detectable at the baseline non-enhanced US (table 2).

The contrast intensity calculations yielded overlapping values for each individual lesion and for each hepatic vascular phase, but on the other hand, all metastatic lesions showed an invariable decrease of their individual intensity values at the sinusoidal-parenchymal liver phase as compared to the corresponding values recorded at the arterial phase. In contrast to this, all three hemangiomas showed a constant increase of the contrast intensity at the sinusoidal-parenchymal liver phase (table 3, fig. 4).
The three-grade scoring (table 1) of the conspicuity of focal lesions for each one of the US techniques yielded a mean value of 2.23 for the non-enhanced US and 2.82 for the CE-US. The difference of the two means was found to be statistically significant $(\mathrm{p}<0.003)$.

\section{Discussion}

Although several US characteristics in focal liver lesions may be related to malignancy and specifically to metastatic disease, considerable overlapping with benign lesion patterns may exist [14]. In addition, the echotexture of both liver metastases and liver parenchyma may be considerably influenced during chemotherapy resulting thus in B-mode US images that are extremely difficult to interpret. The present study shows that CE-US succeeded to diagnose colorectal liver metastases in $17 \%$ more patients and also $17 \%$ more metastases on a per-lesion basis as compared to the B-US. In the same context, the significant scoring differences regarding lesion conspicuity between B-US and CE-US underline the added value from the latter in increasing the diagnostic confidence. Among the 22 patients with focal liver lesions, the conspicuity score for CE-US was reduced (to suboptimal score 2 ) in only 2 patients who showed limited cooperation and presented with lesions at anatomically difficult areas. One other examinee was assigned with a score of 1 in both B-US and CE-US due to heavy hepatic steatosis. The MR imaging study of this particular patient was negative for focal liver lesions. Our results are in accordance with the guidelines of the European Federation of the Societies of Ultrasound in Medicine and Biology (EFSUMB) for the use of CE-US in the follow-up of oncological patients who have liver metastases.

In the present study, a lower rate (17\%) of improved liver metastasis detection was found as opposed to the $46 \%$ reported by Quaia et al. [12]. This difference might be explained by the fact that in the study by Quaia et al., the population under study was mixed including patients suffering from various malignancies and many were imaged before chemotherapy. In the present study all examinees were follow-up colorectal cancer cases at various stages of their chemotherapy regimens. Thus the possibility of a significant remission of preexisting liver metastases should be taken seriously under consideration.

Nonetheless, apart from the issue of lesion detection, CE-US was the technique that yielded the possibility to characterize all detected hepatic lesions with certainty, a task that would have been difficult to be accomplished by 
Fig. 4. A bar graph of contrast intensity values of the liver metastatic lesions and hemangiomas (arrows). Although individual values are overlapping, all metastases show clear reduction of intensity values at sinusoidal-parenchymal liver phase compared to those at the arterial phase. In contrast to this, all hemangiomas show increased values at sinusoidal-parenchymal liver phase.

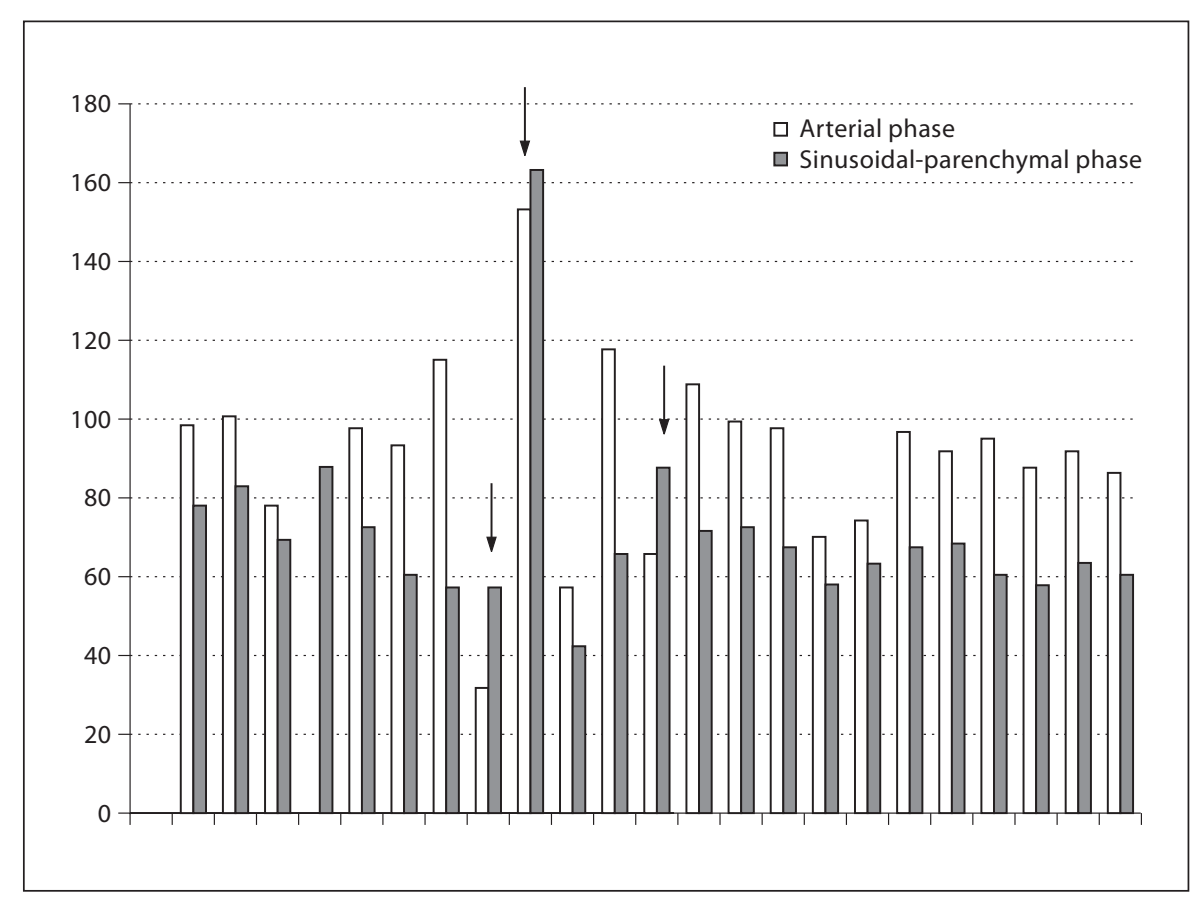

B-US or without the assistance of CT or MR scans. In the present study we have also tried to introduce a quantitative technique in order to enhance the success of focal lesion identification. So far, the attempts to characterize focal liver lesions and hence hepatic metastasis with CEUS rely on a subjective qualitative comparison of the contrast enhancement of lesions against that of the surrounding liver parenchyma [4-10]. By applying direct intensity measurements at specific ROI at the individual focal lesions, we have shown that the expected contrast washout is measurable not only on the hypervascular metastases but also on the colorectal metastases with a reduced arterial supply. In a similar fashion, the hemangiomas yielded a clearly detectable increase of enhancement against time.

The observation that the isoechoic ill-defined metastases in our study population were all hypovascularized during the arterial phase poses the issue that a chemotherapy-induced altered angiogenesis combined with altered echogenicity might exist. It must be pointed out that the well-defined hypoechoic lesions in this study were also hypovascularized at the arterial phase while the target-like hypoechoic with isoechoic center lesions showed predominantly hypervascular arterial phase patterns. Prospective studies with long-term imaging follow-up are needed to clarify the previous observations.
The relatively small study population and the predominance of male patients was the probable reason that we did not detect more rare focal lesions such as focal nodular hyperplasias and hepatic adenomas.

The fact that both observers in this paper were inevitably aware of the existing primary colorectal neoplasia poses a strong potential bias towards the final diagnosis. For this reason a blinded reading of the video recordings by off-site readers should have been the appropriate choice to minimize such a possibility. However, in the present study, the exposure of all the examined individuals to a chemotherapeutic regimen provides a degree of added difficulty at the level of B-US that may have restricted this bias to some extent. Moreover, the inclusion of all examinees in a single group without a reference to preexisting liver lesions or duration of treatment, although a possible limitation as denoted below, provided a further establishment of a 'blinded' approach.

A number of limitations exist in the present study. First, due to the relatively small group of the examined individuals, we did not correlate the pre- and post-contrast echographic patterns of the liver lesions with the histologic type of the colorectal tumor, the site and stage of the primary lesion, the duration of the disease and the type and duration of the chemotherapeutic treatment. Second, we did not perform a CE-US follow-up study to define its capability to illustrate the possible serial altera- 
tions of the patterns of hepatic metastases throughout the treatment. Third, a direct correlation with CE-CT or MR was not feasible since at this point the study was only designed to compare the B-US with CE-US.

In conclusion, the present study has shown that CE-US is able to depict and characterize the colorectal cancer liver metastases with increased rates. Especially in the difficult field of altered liver echogenicity due to chemo- therapy side effects, several inconsistencies that appear with non-enhanced baseline US may be solved efficiently. Quantification of the degree of enhancement adds confidence on the characterization of the metastatic lesions from colorectal cancer. Finally, the observation that the isoechoic ill-defined metastases in our study population were all hypovascularized during arterial phase has to be viewed with larger studies.

\section{References}

1 Cosgrove DO, Bolondi L: Malignant liver disease; in Cosgrove D, Meire H, Dewbury K (eds): Abdominal and General Ultrasound. Edinburgh, Churchill Livingstone, 1993, pp 271-293.

2 Wernecke K, Rummeny E, Bongartz G, et al: Detection of hepatic masses in patients with carcinoma: comparative sensitivities of sonography, CT and MR imaging. AJR Am J Roentgenol 1991;157:731-739.

3 Bleuzen A, Tranquart F: Incidental liver lesions: diagnostic value of cadence contrast pulse sequencing and SonoVue. Eur Radiol 2004;14(suppl 8):P53-P62.

-4 Quaia E, Calliada F, Bertolotto M, et al: Characterization of focal liver lesions by contrast-specific US modes and a sulfur hexafluoride-filled microbubble contrast agent: diagnostic performance and confidence. Radiology 2004;232:420-430.

5 Blomley MJ, Albrecht TA, Cosgrove DO, et al: Improved detection of liver metastases with stimulated acoustic emission in late phase of enhancement with the US contrast agent SH U 508: early experience. Radiology 1999;210:409-416.
6 6 Harvey CJ, Blomley MJ, Eckersley RJ, et al: Hepatic malignancies: improved detection with pulse inversion US in late phase of enhancement with SH U 508 A - early experience. Radiology 2000;216:903-908.

-7 Quaia E, Bertolotto M, Forgács B, et al: Detection of liver metastases by pulse inversion harmonicimaging during levovist late phase: comparison to conventional ultrasound and helical CT in 160 patients. Eur Radiol 2003; 13:475-483.

8 Albrecht T, Blomley MJK, Burns PN, et al: Improved detection of hepatic metastases with pulse-inversion US during the liverspecific phase of SHU 508A: multicenter study. Radiology 2003;227:361-370.

-9 Oldenburg A, Hohmann J, Foert E, et al: Detection of hepatic metastases with low MI real time contrast-enhanced sonography and SonoVue. Ultraschall Med 2005;26:277284.
10 Whittingham T: Contrast-specific imaging techniques: technical perspective; in Quaia E (ed): Contrast Media in Ultrasonography: Basic Principles and Clinical Applications. Berlin, Springer, 2005, pp 43-70.

11 Krix M, Plathow C, Essig M, et al: Monitoring of liver metastases after stereotactic radiotherapy using low-MI contrast-enhanced ultrasound - initial results. Eur Radiol 2005; 15:677-684.

12 Quaia E, D’Onofrio M, Palumbo A, et al: Comparison of contrast-enhanced ultrasonography versus baseline ultrasound and contrast-enhanced computed tomography in metastatic disease of the liver: diagnostic performance and confidence. Eur Radiol DOI 2006;10.1007/s00330-006-0192-7.

13 Hohmann J, Albrecht T, Hoffmann CW, et al: Ultrasonographic detection of focal liver lesions: increased sensitivity and specificity with microbubble contrast agents. Eur J Radiol 2003;46:147-159.

$\checkmark 14$ Harvey CJ, Albrecht T: Ultrasound of focal liver lesions. Eur Radiol 2001;11:1578-1593. 